OPEN ACCESS

Degradation of Proton Exchange Membrane (PEM) Water Electrolysis Cells: Looking Beyond the Cell Voltage Increase

To cite this article: Michel Suermann et al 2019 J. Electrochem. Soc. 166 F645

View the article online for updates and enhancements. 


\title{
Degradation of Proton Exchange Membrane (PEM) Water Electrolysis Cells: Looking Beyond the Cell Voltage Increase
}

\author{
Michel Suermann, $\mathbb{1}^{\mathrm{z}}$ Boris Bensmann, ${ }^{*}$ a and Richard Hanke-Rauschenbach
}

Institute of Electric Power Systems, Leibniz Universität Hannover, 30167 Hannover, Germany

\begin{abstract}
The degradation of proton exchange membrane water electrolysis cells is usually measured in a temporal increase of the cell voltage. Although this is sufficient to evaluate the stability of a system, it is less suitable for targeted material development. Thus, an overpotential-specific and temporally resolved electrochemical characterization protocol is proposed. In this the ohmic overpotential is determined with high frequency resistance measurements. These are also used in combination with polarization curves to distinguish between the kinetic and mass transport overpotentials and to determine kinetic key parameters, according to the Butler-Volmer and transition state theory. Complementary electrochemical impedance spectroscopy measurements further unravel the individual resistances. On this basis, the following statements can already be issued. The major share of the measured cell voltage increase, i.e. degradation, is of apparent nature as it is recovered once lower potentials are applied. It is suggested that this is due to changes in the oxidation states of the iridium-based catalyst. Real degradation occurs in the ohmic and mass transport overpotential mainly at higher current densities and longer operating times. The increasing kinetic overpotential with increasing operating time is primarily potential-driven. Interestingly, both the Tafel slope and the apparent exchange current density slightly increase over time.

(C) The Author(s) 2019. Published by ECS. This is an open access article distributed under the terms of the Creative Commons Attribution 4.0 License (CC BY, http://creativecommons.org/licenses/by/4.0/), which permits unrestricted reuse of the work in any medium, provided the original work is properly cited. [DOI: 10.1149/2.1451910jes]

(cc) BY
\end{abstract}

Manuscript submitted April 5, 2019; revised manuscript received June 17, 2019. Published June 25, 2019.

Proton exchange membrane water electrolysis (PEMWE) systems are currently in the early market introduction phase in the energy sector. At this product's development stage, it is common to focus on durability and reliability rather than on cost. Therefore, evidence has already been provided over a lifetime of several years without measurable degradation, e.g. in the form of a cell voltage increase over operating time. ${ }^{1,2}$ Part of the truth is, however, that such long lifetimes are usually achieved by oversizing the materials used, e.g. by using relatively high catalyst loadings and thick membranes, and by operating the system at moderate conditions in terms of current density, temperature and pressure in order to keep the stressors as low as possible.

On the other hand, however, it offers optimization possibilities with respect to the capital and operating expenditure (CAPEX and OPEX) of a PEMWE system. The catalyst coated membrane (CCM) causes almost one fifth of the stack costs, but promises significant cost reduction possibilities. ${ }^{3,4}$ About half of the OPEX can be attributed to the electricity costs, reflecting the importance of the conversion efficiency. ${ }^{3}$ Thus, one key challenge in current PEMWE technology is the preservation or even increase in durability of the cells while reducing materials cost and increasing conversion efficiency. ${ }^{5}$

However, the stressor-specific degradation mechanisms occurring during electrolysis operation are currently practically not understood, which centrally hinders the evaluation of novel material systems for cell components. Nevertheless there is already a lot of work going on in the field of degradation of PEMWE cell components, as reviewed by Feng et al., ${ }^{6}$ and in the field of the oxygen evolution reaction (OER) catalysts reviewed by Spöri et al. ${ }^{7}$ At the cell level, unfortunately, most of these findings and hypotheses are based on the PEM fuel cell literature and are often transferred to water electrolysis without really considering the different materials, operating and boundary conditions.

Regarding the manner of measuring degradation of PEMWE cells, monitoring the cell voltage (increase) over time seems to be sufficient as long as only the stability of the system is of interest. If, however, a targeted material development and specific operating optimization is aimed at, specific stressor-degradation relations must be known.

In the end, it is aimed to have degradation mechanism-specific accelerated stress tests (ASTs) to reduce the measurement time and costs. While this has been established in the PEM fuel cells community for years, ${ }^{8}$ to date not even a single widely accepted AST protocol exists for PEM water electrolyzers. ${ }^{5}$ Nevertheless, first attempts have been undertaken by several research groups from academia and industry by ramping the current density and hydrogen pressure up and down, ${ }^{9}$ varying current density, ${ }^{10}$ cell temperature and oxygen pressure. ${ }^{11}$ In the aforementioned literature, the experiments are foremost designed to degrade the cell as a whole as fast as possible rather than accelerating component and stressor-specific degradation mechanisms. Furthermore, the degradation was primarily measured in form of a cell voltage increase over time, which accounts for the effect of degradation but does not allow to identify its origin.

Beyond cell voltage monitoring, first attempts to further characterize the electrolysis cell as a function of degradation are performed using current/voltage characteristics (i/E-curves) and electrochemical impedance spectroscopy measurements (EIS), typically at the begin of life (BOL) and end of life (EOL), complemented with ante mortem and post mortem analytical/structural characterization using e.g. scanning electron microscopy (SEM) with energy dispersive X-ray (EDX). ${ }^{12-14}$ Unfortunately, so far, BOL and EOL characterizations provide only an incomplete picture of the ongoing degradation processes.

Consequently, the question arises: what is needed to reveal stressorspecific degradation mechanisms? To the best of our knowledge and belief, i) overpotential-specific degradation rates and ii) time-resolved changes in key parameters would greatly contribute to systematically increase the understanding of the underlying degradation mechanisms.

In this work, we discuss how to tackle at least some of the aforementioned gaps by using different electrochemical characterization methods and data analysis tools. Therefore, the focus is on the proposed approach rather than on the data obtained. The methods and tools are used to unravel the cell voltage increase into time-resolved changes in the main overpotentials combined with monitoring periodically key parameters. The data generated in this way should help to conduct a more targeted material development and optimization of operating conditions.

\section{Experimental}

Test bench and operating conditions.-A commercial PEMWE test bench (Greenlight Innovation, E40) with a constant anode water recirculation flow of $20 \mathrm{ml} /\left(\mathrm{min}^{1} \cdot \mathrm{cm}^{2}\right)$, normalized to the active area, is used. The anode water loop contains an ion exchanger ensuring a water conductivity below or equal to $0.1 \mu \mathrm{S} / \mathrm{cm}$ at any time. The operating temperature and pressure are set to $60^{\circ} \mathrm{C}$ and ambient pressure, respectively.

Electrolysis cell.-A commercial PEMWE test cell (balticFuelCells, quickCONNECT) with an active area of $4 \mathrm{~cm}^{2}$ and a separate 
electric heating for the cell housing is used. The flow fields on the anode as well as on the cathode side are made from titanium (grade 2) and have 13 parallel channels each. In this type of cell, the contact pressure of the active area is set independently of the clamping pressure for the gaskets via a pneumatic actuator. A gas pressure of 2 barg has been applied which corresponds to a contact pressure of about 38 barg according to manufacturer's specification.

Materials.-Commercial catalyst coated membranes (CCMs), based on Nafion 115, with an anode catalyst based on Iridium black with a loading of $2 \mathrm{mg}_{\mathrm{Ir}} / \mathrm{cm}^{2}$ and a cathode catalyst based on Platinum supported on carbon with a loading of $1 \mathrm{mg}_{\mathrm{Pt}} / \mathrm{cm}^{2}$ are used (HIAT). The anode is equipped with a titanium fiber based porous transport layer (PTL) with a nominal thickness of $1 \mathrm{~mm}$, a porosity of $56 \%$ and a fiber diameter of $20 \mu \mathrm{m}$ (Bekaert, titanium grade 1, 2GDL40-1.00). As received from the manufacturer, the PTLs are further used without additional coating and first cleaned before being placed into the cell, as further described below. On the cathode side, a carbon based PTL designed for electrolysis operation, according to manufacturer's specification, with a hydrophobic treatment is used (Freudenberg, H23I2).

Electrochemical measurement equipment and protocols.All experiments are performed with a potentiostat (Bio-Logic, SP-150 with 20 A booster). The PEMWE cells are all investigated according to the measurement scheme shown in Figure 1. The individual steps are explained in detail below.

Preparation.-The titanium PTLs are washed in DI-water in an ultrasonic bath for $15 \mathrm{~min}$ at room temperature. The $\mathrm{CCMs}$, as received from the manufacturer, are immersed in DI-water for one hour at room temperature to ensure a full humidification. ${ }^{15}$ The CCMs are then assembled in wet state in the PEMWE cell and conditioned in the test bench at $60^{\circ} \mathrm{C}$ for another hour by using the recirculating water flow.

Electrochemical characterization: Current/voltage characteristics (i/E-curve). - The PEMWE cells are characterized with i/E-curves from 0.001 to $4 \mathrm{~A} / \mathrm{cm}^{2}$ with logarithmic decimal current density steps. The holding time is 10 seconds plus another 22 seconds for a high frequency resistance (HFR) measurement from 100'000 to $100 \mathrm{~Hz}$ at each current density step. While such short holding times are rather unusual for PEM fuel cells, due to the current density dependent membrane humidification, these are rather unproblematic in electrolysis conditions, as the membrane is always in contact with liquid water. ${ }^{16}$ The HFR is determined by linear interpolation at the intersection of the real part axis in the Nyquist plot, where the imaginary part is equal to zero.

Electrochemical characterization: electrochemical impedance spectroscopy (EIS).- The cells are further characterized with the help of EIS measurements at 0.01, 0.1, 0.2, 0.3, 0.4, 0.6, 0.8, 1, 2, 3 and 4 $\mathrm{A} / \mathrm{cm}^{2}$ from 100 '000 to $0.1 \mathrm{~Hz}$ each. The alternating current is $10 \%$ of the superimposed direct current, as it is used for the aforementioned HFR measurements. While only seven points per decade and one measurement per frequency are used for the HFR measurement at any current density step of the i/E-curve, eleven and three are used for the EIS measurements.

In this work the focus is on the rather well understood HFR and low frequency resistance (LFR), representing the ohmic resistance and total (cell) resistance, respectively. The LFR is determined at the point with the lowest frequency in the first quadrant of the Nyquist plot (typically in the range of 1 to $0.1 \mathrm{~Hz}$ ). It should be noted, that this point is not necessarily the true LFR and thus small deviations from the real cell resistance may occur, especially with progressive operating time and at higher EIS current densities, for which no intersection of the real part axis is obtained. The topic will be discussed in more detail in a separate work.

Electrochemical characterization: current interrupt $(\boldsymbol{C I})$.- - The CI step is performed from a one-minute holding time at $2 \mathrm{~A} / \mathrm{cm}^{2}$ to

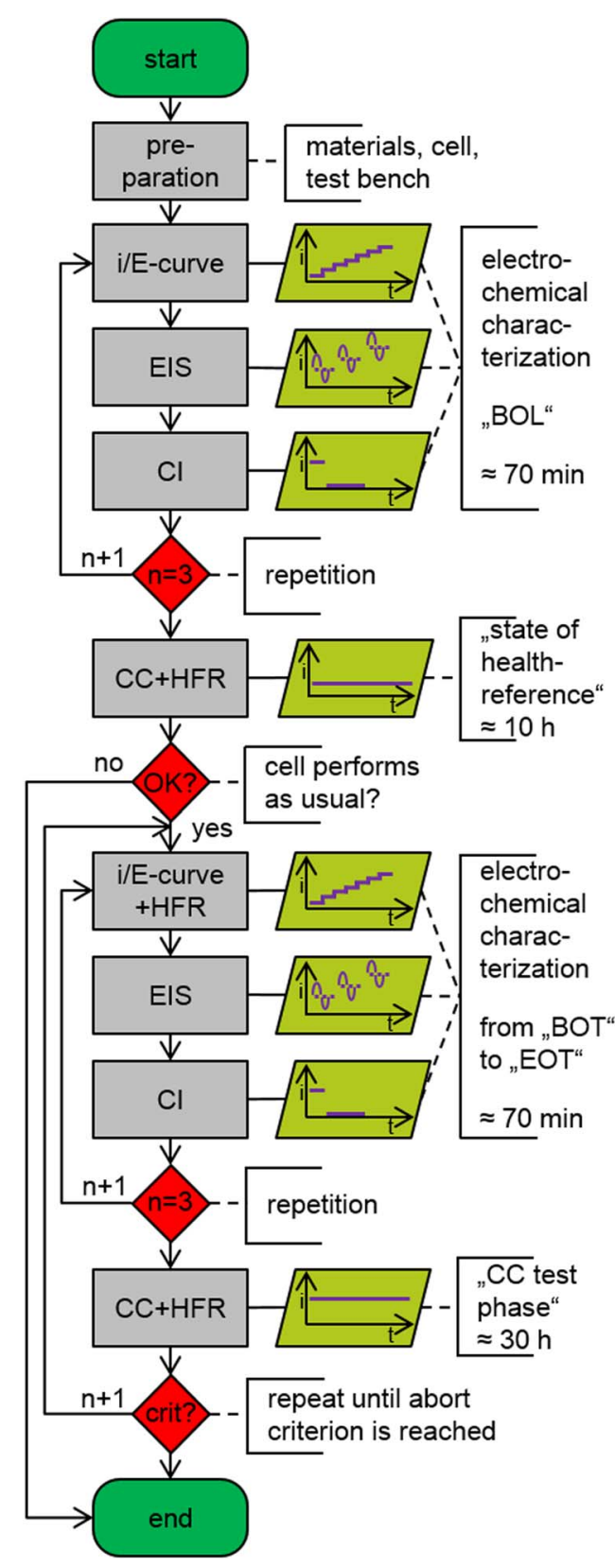

Figure 1. Design of experiment. Abbreviations: $\mathrm{BOL}=$ begin of life; $\mathrm{BOT}=$ begin of test; $\mathrm{EOT}=$ end of test; $\mathrm{CC}=$ constant current; $\mathrm{i} / \mathrm{E}=$ current/voltage; $\mathrm{EIS}=$ electrochemical impedance spectroscopy; $\mathrm{CI}=$ current interrupt.

a five minute holding time at idle or open circuit conditions. Usually this method is used to determine the ohmic resistance, which is, however, not necessary in this work due to the measurement equipment used. Here, only the subsequent relaxation period of five minutes is of interest in order to ensure similar starting conditions for the next step in terms of cell voltage.

The required measurement time for one electrochemical characterization cycle (i/E-curve + EIS + CI) lasts about 70 minutes. Three repetitions are envisaged. All results presented in this work are based on the third run, as no further significant changes are observed after the second run. The changes between the first and the subsequent runs are further discussed in detail later in the context of apparent vs. real degradation.

State of health-reference.-This step is performed at a constant current density of $1 \mathrm{~A} / \mathrm{cm}^{2}$ combined with an HFR measurement 
similar to before, but every 15 minutes. The reference step is preliminarily used for internal benchmarking. After this step it is decided whether the cell performs as usual or not. Thus, problems due to e.g. contamination, material defects or incorrect assembling are recognized early and misinterpretations are minimized later on.

Constant current $(\boldsymbol{C C})$ test phase.-This is the only step of the measurement protocol that differs from cell to cell. For proof of concept, in this work, two different constant current densities, i.e. 1 and $4 \mathrm{~A} / \mathrm{cm}^{2}$, are investigated. Similar to the reference step also here the HFR is measured each 15 minutes. The measurement time of each $\mathrm{CC}$ test phase is about 30 hours. Each cell has been measured for at least eight repetitions, corresponding to a total measurement time of at least $270 \mathrm{~h}$. The aforementioned parameters such as constant current density and cycle duration are selected as a result of preliminary tests, but are not discussed further here, as the focus of this paper is on the electrochemical methods and analysis tools.

The end of each test is chosen for test bench capacity reasons rather than for relevant safety or degradation issues, as can be seen from the results discussed below. Since a two electrode setup is used, the cell voltages are reported versus the reversible hydrogen electrode (RHE). Therefore, it is assumed that the $\mathrm{H}^{+}$- activity in the polymer electrolyte and the pressure of $\mathrm{H}_{2}$ are in equilibrium at the Pt-cathode.

\section{Results and Discussion}

The results section is structured analogously to the design of experiment sketched in Figure 1. As a starting point the results of the $30 \mathrm{~h} \mathrm{CC}$ test phase are discussed.

Information gathered during constant current test phase.-Usually PEMWE degradation experiments are carried out at a constant current density and the cell voltage increase over time is monitored. The corresponding degradation rate is given in $\mu \mathrm{V} / \mathrm{h}$, and it is in the single-digit to lower double-digit range, at least for commercial systems. However, such relatively low values are typically only achieved at moderate operating conditions with respect to current density/cell voltage, temperature and pressure and by using oversized materials. The materials investigated here can also rather be seen as oversized. The operating conditions are also rather moderate, i.e. $60^{\circ} \mathrm{C}$ and ambient pressure. The only exception is the relatively high current density of $4 \mathrm{~A} / \mathrm{cm}^{2}$ compared to the rather moderate $1 \mathrm{~A} / \mathrm{cm}^{2}$.

Indeed, when only the stability or durability of the system is of interest, monitoring the cell voltage increase over time seems to be sufficient. Such an approach is shown in Figure 2a for the two cells investigated at 1 and $4 \mathrm{~A} / \mathrm{cm}^{2}$. The only difference to a classical approach is that here the constant current density is hold for "only" 30 hours in one piece, followed each by the electrochemical characterization part (i/E-curve, EIS, CI). In each $30 \mathrm{~h} \mathrm{CC}$ test cycle the cell voltage increases over time, especially in the very beginning of each cycle. With respect to the electrochemical characterization period in between two $30 \mathrm{~h}$ cycles, it can already be determined with the naked eye that beneficial processes mainly offset the cell voltage increase during the $30 \mathrm{~h} \mathrm{CC}$ test phase. Such behavior is frequently observed in literature, ${ }^{12,13}$ but is usually not considered in the data analysis. This offset phenomenon is hereinafter referred to as apparent degradation, whereas the irretrievable cell voltage increase is referred to as real degradation.

The observed cell voltage increase over time is further converted to a degradation rate and shown in Figure $2 b$. It should be noted that the degradation rate is calculated from one to the next start value per $30 \mathrm{~h}$ $\mathrm{CC}$ test phase. The same applies to the end values. The start and end values typically correspond to the minimum and maximum cell voltage values of each $30 \mathrm{~h} \mathrm{CC}$ test phase, as highlighted with larger symbols in Figure 2a. Obvious and little surprising the degradation rate of the $4 \mathrm{~A} / \mathrm{cm}^{2}$ sample is with about $200+\mu \mathrm{V} / \mathrm{h}$ higher than those of the $1 \mathrm{~A} / \mathrm{cm}^{2}$ sample with $10 \pm 30 \mu \mathrm{V} / \mathrm{h}$. Interestingly, both the degradation rates related to the end/maxima and to some extent to the start/minima values are quite stable over time, except for the $4 \mathrm{~A} / \mathrm{cm}^{2}$ cell in the first
150 hours. While the degradation rate based on the start values can somehow be considered as the real degradation, the end values include parts of apparent degradation effects of varying intensity. The apparent degradation refers to the difference between the end and start values directly before and after an electrochemical characterization phase. The corresponding degradation rate is typically an order of magnitude greater than that for the start and end values.

Nevertheless, at this point it can honestly only be concluded that both cells degrade with a fairly constant degradation rate, which is significantly higher for the $4 \mathrm{~A} / \mathrm{cm}^{2}$ than for the $1 \mathrm{~A} / \mathrm{cm}^{2}$ sample. However, the question of the origin of the degradation effect cannot be answered this way.

Consequently, next to the cell voltage monitoring also the HFR is measured periodically. With the help of the HFR measurement the ohmic overpotential is determined. At the same time, also the iRfree cell voltage is obtained, which merges the dominant kinetic and minor mass transport losses. Analogous to the cell voltage, also the temporal progressions of the ohmic overpotential and the iR-free cell voltage are shown in Figures 2c/2e), as well as the corresponding degradation rates in Figures $2 \mathrm{~d} / 2 \mathrm{f}$. It can be highlighted that the aforementioned apparent degradation phenomena appear mainly for the $4 \mathrm{~A} / \mathrm{cm}^{2}$ sample and almost entirely for the $1 \mathrm{~A} / \mathrm{cm}^{2}$ sample in the iR-free cell voltage, indicating changes in the OER kinetics.

As a small excursus: In a further separate test it is shown that relatively short holding times of one minute at low cell voltages, but still above $1.48 \mathrm{~V}$, are sufficient to recover most of the apparent degradation effect. The following applies: the lower the cell polarization, the greater the effect. It can be assumed that this is directly related to the oxidation state of the $\mathrm{IrO}_{\mathrm{x}}$-based catalyst, which is reduced with the hydrogen gas crossover. ${ }^{17-20}$ If the Iridium oxide-based catalyst is operated too long/too often well below the electrolysis cell voltage toward $0 \mathrm{~V}$ vs RHE, this can even lead to increased degradation rates. ${ }^{20}$ For further details, please have a look at the supplementary material including the results of the complementary experiment.

Nevertheless, with respect to the degradation rates representing the real degradation, however, interestingly for both cells very similar $i R$-free cell voltage degradation rates are obtained in the mid-doubledigit range (in $\mu \mathrm{V} / \mathrm{h}$ ), with greater fluctuations occurring for the 4 $\mathrm{A} / \mathrm{cm}^{2}$ sample. While for the $1 \mathrm{~A} / \mathrm{cm}^{2}$ sample these values almost entirely fit to the aforementioned degradation rate with respect to the cell voltage (compare to Figure $2 \mathrm{~b}$ ), it represents only a small share of it for the $4 \mathrm{~A} / \mathrm{cm}^{2}$ sample. Consequently, for the $4 \mathrm{~A} / \mathrm{cm}^{2}$ sample the major part of the real degradation is observed in the ohmic overpotential, as shown in Figure 2d.

At this point a much more differentiated conclusion can be drawn with the help of a simple HFR measurement than without. The major part of the apparent degradation effect is matched to the iR-free cell voltage, reflecting changes in the OER kinetics. Furthermore, similar real iR-free cell voltage degradation rates are observed for both cells, which in turn suggests similar degradation processes. Unfortunately, even with this information a targeted material-optimization is afar

Information gathered from current/voltage-characteristics (i/Ecurve).- In the previous section, for both cells a relatively large part of the observed degradation, mainly in the iR-free cell voltage, is of apparent nature. Now the focus should be on the remaining losses of real nature. Therefore, we will now devote ourselves to the electrochemical characterization part, starting with the $\mathrm{i} / \mathrm{E}$-curves. The presented data are comparable to the start values of each $30 \mathrm{~h} \mathrm{CC}$ test phase shown before in Figure 2e.

In Figure $3 a$ the iR-free polarization curves for both cells are shown in a Tafel-plot, exemplarily at the begin of test (BOT) and after $240 \mathrm{~h}$ measurement time (compare to Figure 1). The iR-free cell voltage difference between the $240 \mathrm{~h}$ and the BOT curves is exposed separately in Figure 3b. It can be highlighted that even at relatively small current densities an increase of about 4 and $8 \mathrm{mV}$ is noticed for the 1 and $4 \mathrm{~A} / \mathrm{cm}^{2}$ sample, respectively. At higher current densities, above about $0.1 \mathrm{~A} / \mathrm{cm}^{2}$ significantly higher deviations are noticed each, especially 

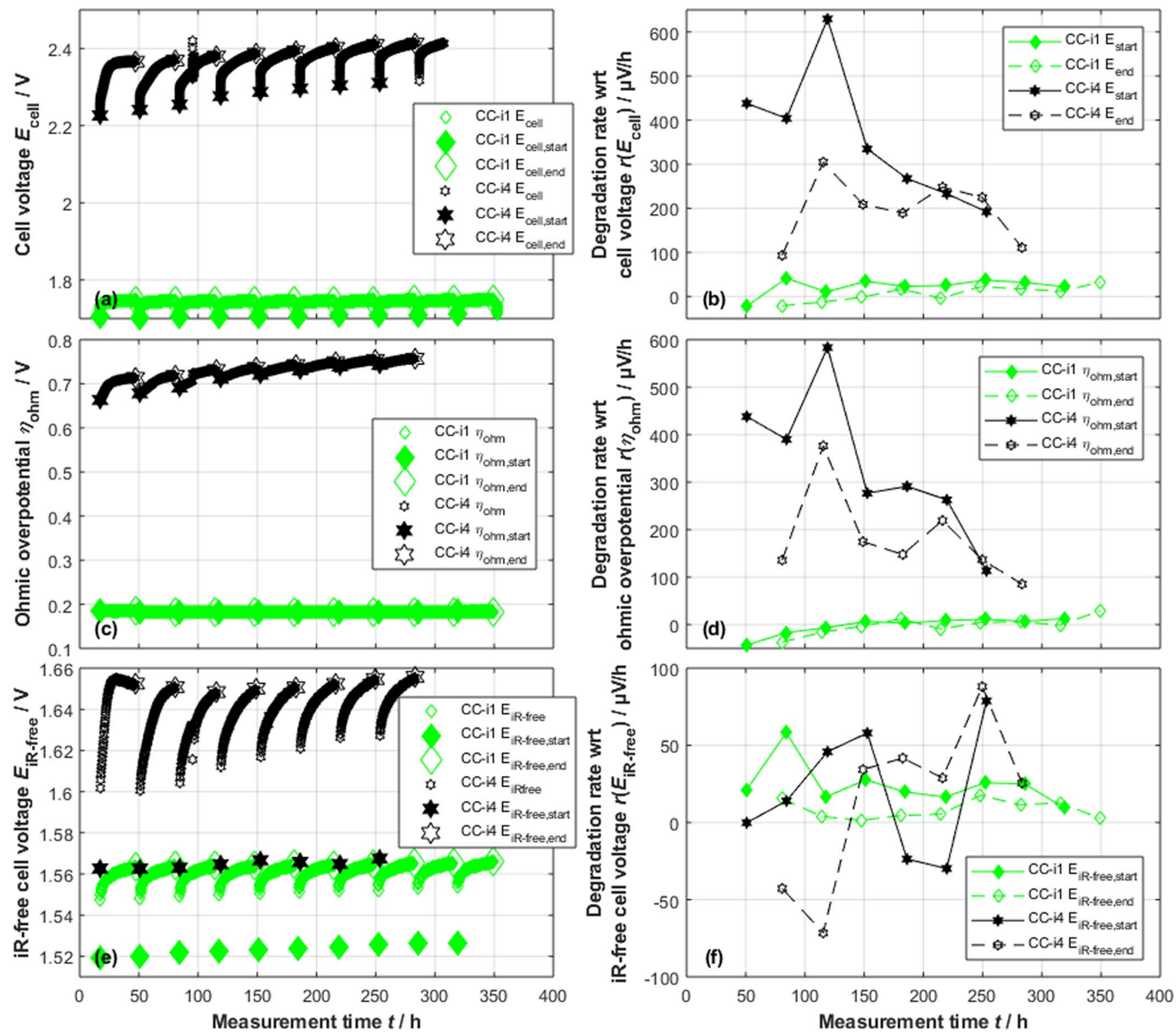

Figure 2. (a) The measured cell voltage, (c) the ohmic overpotential and (e) the iR-free cell voltage of the $30 \mathrm{~h} \mathrm{CC}$ test cycles are shown as a function of the measurement time. The measurement values at the start and end time of each $30 \mathrm{~h}$ measurement period are highlighted with larger symbols and further used to calculate the corresponding degradation rates shown in (b), (d) and (f), respectively, as described in the text.

for the $4 \mathrm{~A} / \mathrm{cm}^{2}$ sample, which are allocated to increasing mass transport losses.

The iR-free cell voltage changes at relatively small current densities indicate variations in catalyst-specific parameters such as transfer coefficient, reaction order, activation energy and exchange current density. ${ }^{21}$ Thus, an overpotential analysis, based on the Butler-Volmer and transition state theory, ${ }^{22}$ is performed as follows. A Tafel fit is applied in the linear range of the $\mathrm{i} / \mathrm{E}$-curves between 0.01 and $0.1 \mathrm{~A} / \mathrm{cm}^{2}$, where mass transport losses are assumed to be negligible. Since the rate determining step is rather part of the sluggish OER, than the quasireversible hydrogen evolution reaction (HER), ${ }^{23}$ the corresponding kinetic parameters are assigned to the OER. For further information on the aforementioned analysis method, we refer to previous work. ${ }^{16,24}$

The corresponding changes of the Tafel slopes at any electrochemical characterization cycle are shown in Figure 4a. By extrapolating the Tafel line toward equilibrium conditions, i.e. $\eta_{\mathrm{OER}}=0 \mathrm{~V}$, the apparent exchange current density is determined. Here a thermodynamic cell voltage of $1.1948 \mathrm{~V}$ is calculated with the Nernst equation (at $60^{\circ} \mathrm{C}$ and ambient pressure), assuming ideal gas behavior and an activity of unity for the liquid water. ${ }^{25-27}$ Both the Tafel slope and the apparent exchange current density slightly increase over the measurement time, as presented in Figure 4. While an increase of the Tafel slope is rather disadvantageous with respect to the corresponding overpotential, an increase of the apparent exchange current density is advantageous. Consequently, due to the opposing trends, only a relatively small increase in the iR-free cell voltage is observed, as already shown in Figure 2f. Consequently, the stressor seems to be mainly the electrode polarization, which is in the same magnitude for both investigated cells, rather than the current density.

It should be noted, however, that the observed changes in the Tafel slope and apparent exchange current density are rather small, but the same applies to the measurement time when comparing to commercial life times. It would therefore be very interesting to observe these parameters over a period of months to years. It should also be verified how far the assumption of the mass transport loss-free region below $0.1 \mathrm{~A} / \mathrm{cm}^{2}$ is correct, since variations with the degree of degradation, e.g. in the proton conductivity ${ }^{28,29}$ or in the current density-dependent gas permeation of the ionomer in the catalyst layer ${ }^{30}$ are conceivable. 

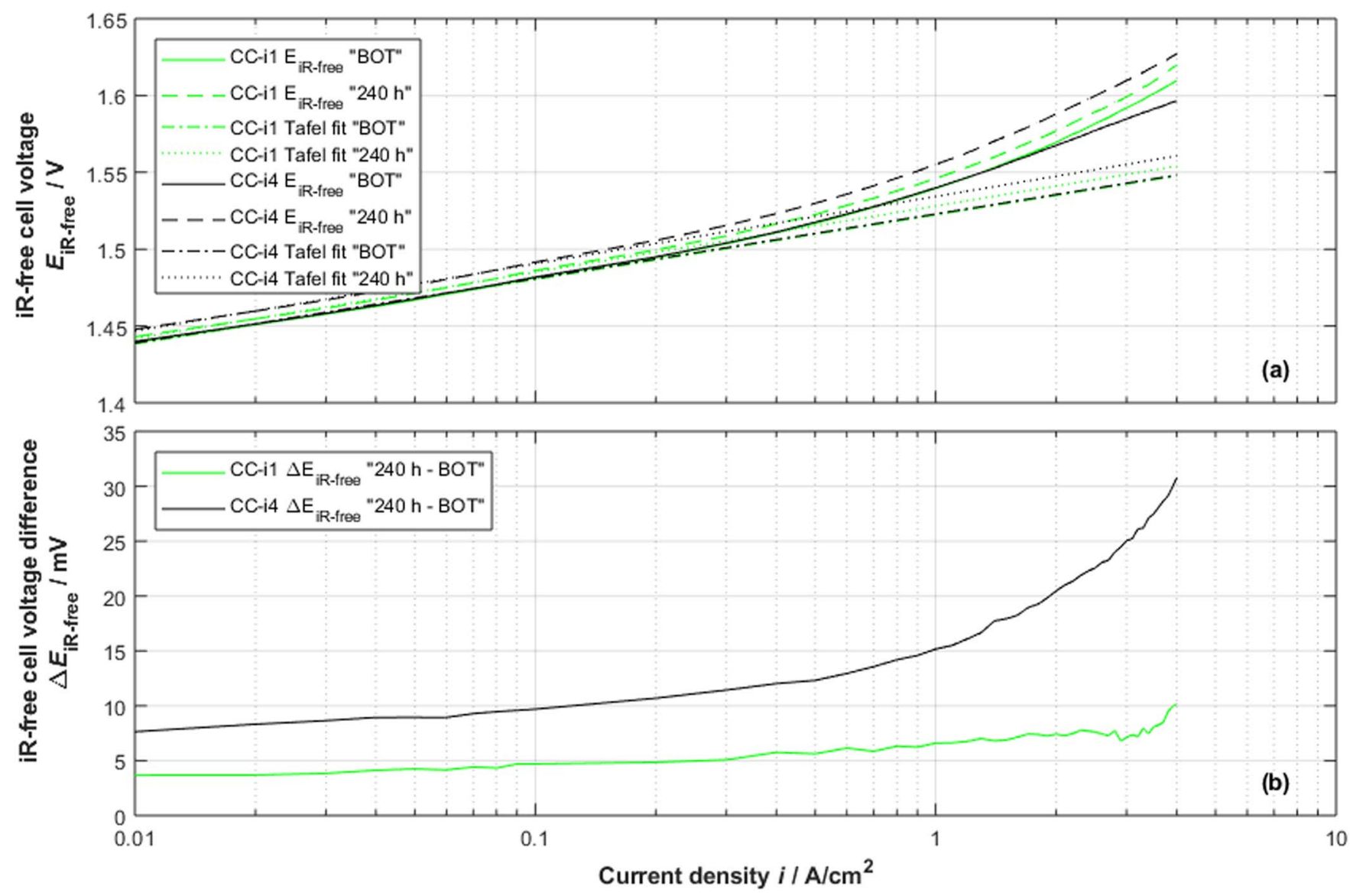

Figure 3. (a) Tafel plots of the iR-free cell voltage with corresponding Tafel lines for the 1 and $4 \mathrm{~A} / \mathrm{cm}^{2} \mathrm{CC}$ test phase samples at BOT and after $240 \mathrm{~h}$ operating time. (b) iR-free cell voltage difference between the $240 \mathrm{~h}$ and the BOT i/E-curve.

At this point, and by the help of i/E-curves combined with HFR measurements, it is possible to further reveal the degradation effects. On the one hand, the iR-free cell voltage increase is caused by a deterioration in the OER reaction kinetics and especially for the $4 \mathrm{~A} / \mathrm{cm}^{2}$
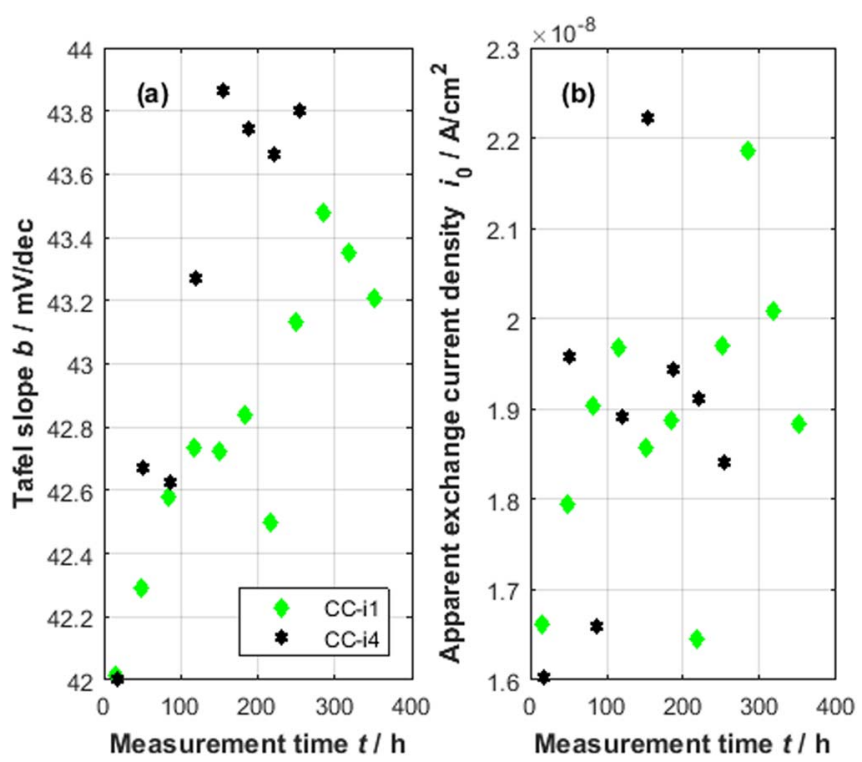

Figure 4. (a) Tafel slope $b$ and (b) apparent exchange current density $i_{0}$ as a function of the measurement time for the different samples as indicated. cell by an increase in the mass transport losses. It should be noted that regarding the kinetics, both the Tafel slope and the apparent exchange current density increase over time, resulting in a partly offset of the resulting OER overpotential, as those processes are opposed to each other.

Information gathered from electrochemical impedance spectroscopy (EIS) measurements.-As shown in the previous section, with the help of the $\mathrm{i} / \mathrm{E}$-curves an increase in the kinetic and mass transport overpotential with increasing operating time, especially for the $4 \mathrm{~A} / \mathrm{cm}^{2}$ sample, is highlighted. To double check and to better understand the ongoing degradation effects, EIS measurements are performed from $100^{\prime} 000$ to $0.1 \mathrm{~Hz}$ at different current densities. As the interpretation of the capacitive and especially inductive phenomena at low frequencies are still under discussion and often inconsistently interpreted, as discussed in Ref. 16, in this study the focus is on the rather well understood high frequency and low frequency resistance, representing the ohmic resistance and total cell resistance, respectively. In Figure 5 the HFR and the difference between the LFR and HFR are shown exemplarily for EIS measurement current densities of 0.1, 0.4, 1 and $4 \mathrm{~A} / \mathrm{cm}^{2}$. It should be noted, that there is a discrepancy between the BOT area specific HFR values of the two cells of about $15 \mathrm{~m} \Omega \cdot \mathrm{cm}^{2}$ or $\sim 8 \%$, which is associated to the material and cell/measuring setup. It is therefore more appropriate to take into account the respective changes.

While the HFR stays almost constant for the $1 \mathrm{~A} / \mathrm{cm}^{2}$ sample, at least up to EIS current densities of about $1 \mathrm{~A} / \mathrm{cm}^{2}$, an increase of about $5 \mathrm{~m} \Omega \cdot \mathrm{cm}^{2}$ per $100 \mathrm{~h}$ is measured for the $4 \mathrm{~A} / \mathrm{cm}^{2}$ sample at any EIS current density (Figures $5 \mathrm{a}-5 \mathrm{~d}$ ). The increasing HFR could be caused by cationic contamination of the membrane, dissolution and re-precipitation of the Iridium within the membrane or rather by an 

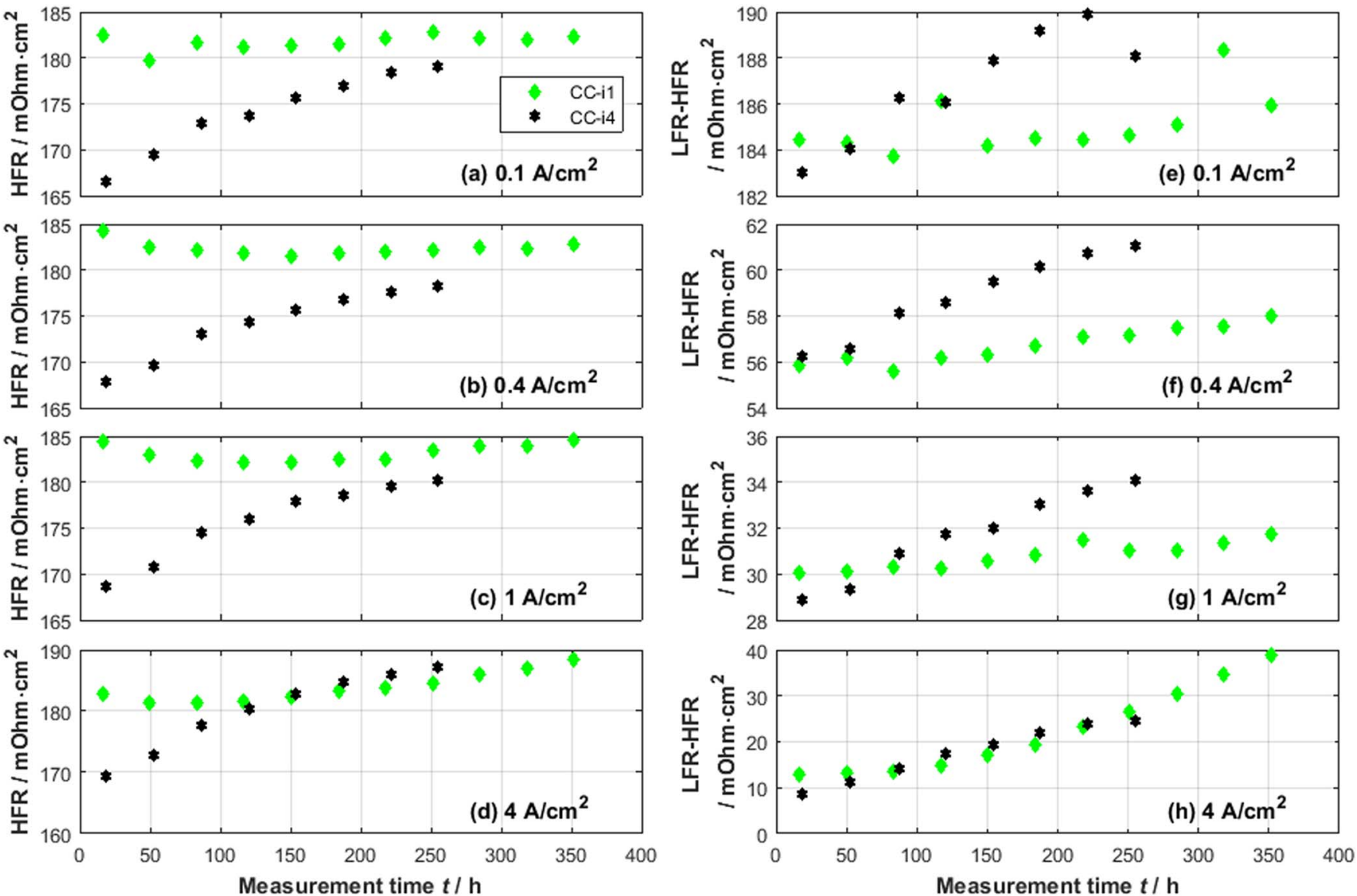

Figure 5. (a-d) HFR versus measurement time at different EIS measurement current densities as indicated. (e-h) LFR-HFR versus measurement time at different EIS measurement current densities.

increase in the interfacial contact resistances between the titanium PTL and the anode and/or the flow field, as recently discussed by Weiß et al. ${ }^{20}$ With respect to the difference between the LFR and HFR (Figures $5 \mathrm{e}-5 \mathrm{~h}$ ), a similar trend is observed as before until EIS current densities below or equal to $1 \mathrm{~A} / \mathrm{cm}^{2}$. While for the $4 \mathrm{~A} / \mathrm{cm}^{2}$ sample an increase of about $2 \mathrm{~m} \Omega \cdot \mathrm{cm}^{2}$ per $100 \mathrm{~h}$ is measured, it is significantly lower with about $0.5 \mathrm{~m} \Omega \cdot \mathrm{cm}^{2}$ per $100 \mathrm{~h}$ for the $1 \mathrm{~A} / \mathrm{cm}^{2} \mathrm{sam}-$ ple. Surprisingly, at higher EIS current densities, however, a similar degradation is measured, e.g. about $8 \mathrm{~m} \Omega \cdot \mathrm{cm}^{2}$ per $100 \mathrm{~h}$ at $4 \mathrm{~A} / \mathrm{cm}^{2}$ (Figure 5h).

At this point, a clearer differentiation of the occurring degradation effect can be made by using EIS measurements at different current densities. Regarding the HFR and similar to the observations made from the $30 \mathrm{~h} \mathrm{CC}$ test phases, a continuous increase could only be noticed for the $4 \mathrm{~A} / \mathrm{cm}^{2}$ sample. Regarding the difference between the LFR and HFR, an increase with a factor of four from the 1 to the $4 \mathrm{~A} / \mathrm{cm}^{2}$ sample is observed with EIS measurements below and equal to about $1 \mathrm{~A} / \mathrm{cm}^{2}$. Somewhat surprisingly, a very similar and significantly higher degradation rate of about $8 \mathrm{~m} \Omega \cdot \mathrm{cm}^{2}$ per $100 \mathrm{~h}$ is measured at higher EIS measurement current densities, indicating a similar degradation mechanism for the two cells. In other words, similar degradation effects are observed only at higher EIS measurement current densities, although the constant current density varies greatly during the relatively long $30 \mathrm{~h}$ test phases.

Summarizing, if only the long-term stability of the PEMWE system is of interest, it seems to be more than sufficient to monitor only the cell voltage (increase) over time. However, if the understanding of the occurring degradation effects, phenomena and mechanisms are of interest, supplementary, at best non-invasive, electrochemical methods appear to be essential. At the top of the list, a simple but powerful HFR measurement already allows to differentiate between the main overpotentials, i.e. kinetic, ohmic and mass transport. In the next place, simple polarization curves, again combined with HFR measurements at its best, allow to further differentiate between kinetic and mass transport overpotentials in the entire current density range. Furthermore, and according to the Butler-Volmer and transition state theory, changes in catalyst-specific kinetic parameters such as Tafel slope and apparent exchange current density can be determined easily. Last but not least complementary EIS measurements allow to better confine the origin of degradation due to the specific time constants.

This is only a foretaste of what would be possible when studying the degradation of PEMWE cells and, in our view, what would be necessary in order to reveal stressor-specific degradation mechanisms. It is only on the basis of these principles, that it is possible to derive corresponding ASTs and to ultimately advance a targeted material development.

Further remark.-Finally, we would like to draw the reader's attention to the previously used unit of degradation rate, i.e. $\mu \mathrm{V} / \mathrm{h}$. As in most use cases the amount of hydrogen produced is of greater interest than the operating time, the degradation rates previously shown in Figure $2 b$ are normalized to the area-specific produced amount of hydrogen, according to the Faraday's law, and are shown in Figure 6. For the sake of simplicity, additional losses such as gas crossover and leakages are neglected, as are the total energy consumption and time dependence of the degradation. Little surprising, the degradation rates of the 1 and $4 \mathrm{~A} / \mathrm{cm}^{2}$ samples come close together, due to the different current densities by the factor of four. This circumstance is also evident when looking again on the aforementioned results, e.g. the differences between the LFR and HFR (Figures 5e-5g), where also a factor of about four is noticed for EIS measurement current densities up to $1 \mathrm{~A} / \mathrm{cm}^{2}$. Consequently, when normalizing the degradation 


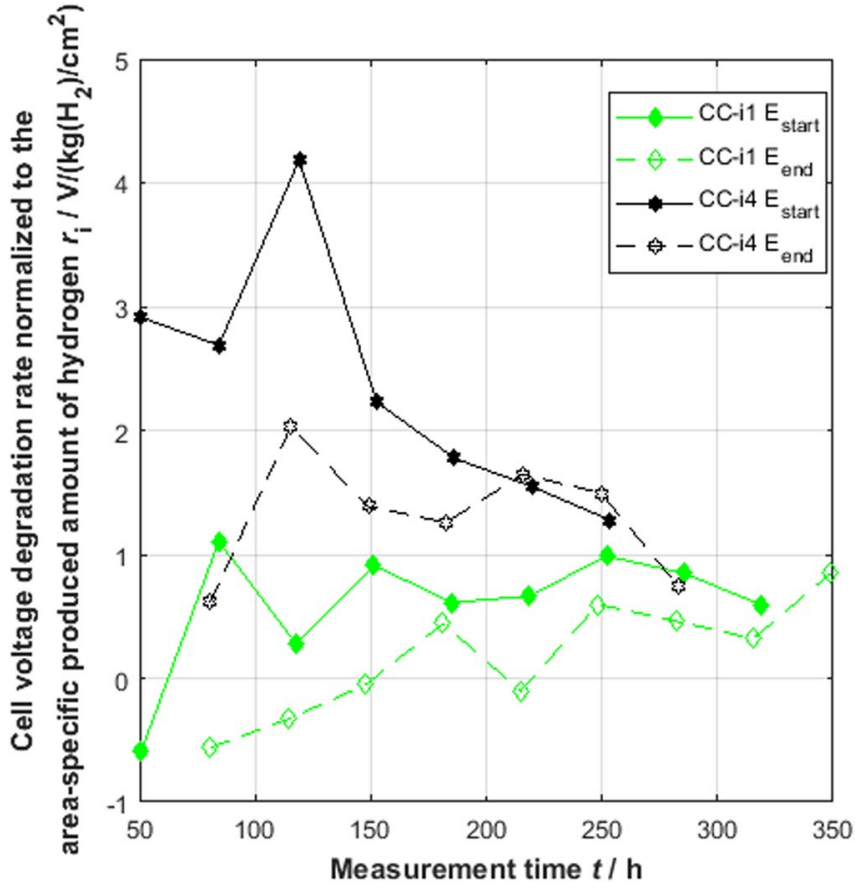

Figure 6. The cell voltage degradation rate normalized to the area-specific produced amount of hydrogen.

rates to the produced amount of hydrogen, the applied current density seems to be less sensitive than before.

\section{Conclusions}

In this work, relatively simple but powerful electrochemical measurement methods and data analysis tools are presented and combined in order to move closer to identify stressor-specific degradation mechanisms. This is considered necessary to derive accelerated stress tests and to conduct a targeted material development in the end. The focus here is clearly on the proposed approach and points out possibilities for determining key parameters with the aid of different electrochemical measurement methods. These parameters can then be used later in further series of measurements to better describe the effect of degradation on the cells.

During constant current test phases the largest part of the cell voltage increase is of apparent nature and is assigned to changes in the oxidation states of the Iridium oxide-based anode catalyst.

The origin of the real degradation could already be assigned to the iR-free cell voltage for both cells to a very similar extent with the help of high frequency resistance measurements. While these losses account for almost the entire losses for the $1 \mathrm{~A} / \mathrm{cm}^{2}$ sample, the $4 \mathrm{~A} / \mathrm{cm}^{2}$ sample suffered significantly larger losses in the ohmic overpotential.

Complementary polarization curves showed that, even at very small current densities, an increase in the iR-free cell voltage, representing the kinetic overpotential, is obtained. This deviation from the state of health at begin of test increased significantly with higher current densities and progressing measurement time. Thus, next to an increase in the kinetic overpotential, also an increase in the mass transport overpotential is observed.

The change in the kinetic overpotential could be explained by increasing Tafel slopes and increasing apparent exchange current densities. It should be noted, however, that the latter partly offsets the resulting overpotential.

By the help of electrochemical impedance spectroscopy measurements an almost steady increase in the high frequency resistance and in the iR-free cell resistance could be noticed for the $4 \mathrm{~A} / \mathrm{cm}^{2}$ sample at any EIS current density. Surprisingly, a similar trend is observed only for the $1 \mathrm{~A} / \mathrm{cm}^{2}$ sample for EIS current densities higher than the constant current density during the long measurement phase, i.e. above $1 \mathrm{~A} / \mathrm{cm}^{2}$.

It is planned, to extend the introduced and discussed electrochemical methods and to systematically study different stressor and material combinations. Complementary analytical investigation at ante mortem, post mortem and at different state of health times should provide a significantly better database and thus understanding. Ultimately, it is planned to process this information in a model-theoretical approach to be able to reveal the underlying fundamental mechanisms and reactions in order to be able to determine and even forecast the state of health of a PEM water electrolyzer and to develop degradation mechanism-specific accelerated stress tests.

\section{Acknowledgments}

The authors gratefully acknowledge the financial support by the Federal Ministry of Education and Research of Germany in the framework of PowerMEE (project number 03SF0536B).

\section{ORCID}

Michel Suermann (D) https://orcid.org/0000-0001-9685-7081 Boris Bensmann (D) https://orcid.org/0000-0001-8685-7192

\section{References}

1. K. E. Ayers, C. Capuano, and E. B. Anderson, ECS Transactions, 41(10), 15 (2012)

2. K. E. Ayers, J. N. Renner, N. Danilovic, J. X. Wang, Y. Zhang, R. Maric, and H. Yu, Catalysis Today, 262, 121 (2016)

3. L. Bertuccioli, A. Chan, D. Hart, F. Lehner, B. Madden, and E. Standen, Study on development of water electrolysis in the EU, (2014).

4. K. E. Ayers, E. B. Anderson, C. Capuano, B. Carter, L. Dalton, G. Hanlon, J. Manco, and M. Niedzwiecki, ECS Transactions, 33(1), 3 (2010).

5. U. Babic, M. Suermann, F. N. Büchi, L. Gubler, and T. J. Schmidt, J. Electrochem. Soc., 164(4), F387 (2017).

6. Q. Feng, X. Yuan, G. Liu, B. Wei, Z. Zhang, H. Li, and H. Wang, Journal of Power Sources, 366, 33 (2017).

7. C. Spöri, J. T. H. Kwan, A. Bonakdarpour, D. P. Wilkinson, and P. Strasser, Angewandte Chemie (International ed. in English), 56(22), 5994 (2017).

8. U.S. Department of Energy (DOE), U.S. DRIVE Fuel Cell Tech Team, Cell Compo nent Accelerated Stress Test and Polarization Curve Protocols for PEM Fuel Cells, (2013).

9. K. E. Ayers, E. Anderson, C. Capuano, G. Hanlon, and E. Styche, 215th ECS Meeting (Abstract \#380) (2009).

10. T. Lickert, C. Schwarz, P. Gese, A. Fallisch, M. Schlüter, N. Höfling, and T. Smolinka, Towards selective test protocols for accelerated Towards selective test protocols for accelerated in situ degradation of PEM electrolysis cell components, (2017).

11. F. van Berkel, A. de Groot, and S. ten Hoopen, Impact of Dynamic Load from Renewable Energy Sources on PEM Electrolyzer Lifetime, (2017).

12. S. Siracusano, N. van Dijk, R. Backhouse, L. Merlo, V. Baglio, and A. S. Aricò, Renewable Energy, 123, 52 (2018).

13. C. Rakousky, G. P. Keeley, K. Wippermann, M. Carmo, and D. Stolten, Electrochimica Acta, 278, 324 (2018)

14. F. Fouda-Onana, M. Chandesris, V. Médeau, S. Chelghoum, D. Thoby, and N. Guillet, International Journal of Hydrogen Energy, 41(38), 16627 (2016).

15. G. Alberti, R. Narducci, and M. Sganappa, Journal of Power Sources, 178(2), 575 (2008).

16. M. Suermann, T. J. Schmidt, and F. N. Büchi, Electrochimica Acta, 211, 989 (2016).

17. P. J. Rheinländer, M. Bernt, Y. Incedag, and H. A. Gasteiger, PRiME 2016/230th ECS Meeting, Abstract \#2427 (2016).

18. S. Geiger, O. Kasian, M. Ledendecker, E. Pizzutilo, A. M. Mingers, W. T. Fu, O. Diaz-Morales, Z. Li, T. Oellers, L. Fruchter, A. Ludwig, K. J. J. Mayrhofer, M. T. M. Koper, and S. Cherevko, Nat Catal, 1(7), 508 (2018).

19. V. Pfeifer, T. E. Jones, J. J. Velasco Vélez, R. Arrigo, S. Piccinin, M. Hävecker, A. Knop-Gericke, and R. Schlögl, Chemical science, 8(3), 2143 (2017)

20. A. Weiß, A. Siebel, M. Bernt, T.-H. Shen, V. Tileli, and H. A. Gasteiger, J. Elec trochem. Soc., 166(8), F487 (2019).

21. K. C. Neyerlin, W. Gu, J. Jorne, and H. A. Gasteiger, J. Electrochem. Soc., 153(10), A1955 (2006)

22. J. O.'M. Bockris and A. K. N. Reddy, Modern electrochemistry, New York, Kluwer Academic Publishers (2002)

23. J. Durst, A. Siebel, C. Simon, F. Hasché, J. Herranz, and H. A. Gasteiger, Energy Environ Sci, 7(7), 2255 (2014).

24. M. Suermann, T. J. Schmidt, and F. N. Büchi, Electrochimica Acta, 281, 466 (2018).

25. I. Barin, Thermochemical data of pure substances, Weinheim, VCH (1995).

26. R. Wiebe and V. L. Gaddy, J. Am. Chem. Soc., 56(1), 76 (1934). 
27. D. Tromans, Hydrometallurgy, 48(3), 327 (1998).

28. U. Babic, E. Nilsson, A. Pătru, T. J. Schmidt, and L. Gubler, J. Electrochem. Soc., 166(4), F214 (2019)
29. M. Bernt and H. A. Gasteiger, J. Electrochem. Soc., 163(11), F3179 (2016).

30. P. Trinke, G. P. Keeley, M. Carmo, B. Bensmann, and R. Hanke-Rauschenbach, $J$. Electrochem. Soc., 166(8), F465 (2019). 